\title{
CONDITIONS FOR A UNIVERSAL MAPPING OF ALGEBRAS TO BE A MONOMORPHISM
}

\author{
BY JOHN L. MACDONALD
}

Communicated by Saunders Mac Lane, January 3, 1974

Introduction. We give necessary and sufficient conditions for the monicity of any given unit morphism associated with an algebraic functor and its left adjoint. The direct verification of these conditions in specific cases can be somewhat subtle. However, a stronger set of sufficient conditions for monicity may be given which is easier to check directly. The latter conditions are still sufficiently general to provide a categorical form for the proof of the Birkhoff Witt theorem, closely related to Birkhoff's original proof [3], as well as one for the Schreier theorem on free products of groups with amalgamated subgroups [1], [5].

1. Necessary and sufficient conditions. We consider algebras defined by a set $\Omega$ of operators and a set $E$ of identities as in Mac Lane [4]. The diagram $V U: \mathscr{A} \rightarrow \mathscr{B} \rightarrow \mathscr{D}$ is called a standard diagram of algebras if

1. $\mathscr{A}, \mathscr{B}$ and $\mathscr{D}$ are the categories of $\langle\Omega, E\rangle,\left\langle\Omega^{\prime}, E^{\prime}\right\rangle$ and $\left\langle\Omega^{\prime \prime}, E^{\prime \prime}\right\rangle$ algebras, respectively, with $\Omega^{\prime \prime} \subseteq \Omega^{\prime}$ and $E^{\prime \prime} \subseteq E^{\prime}$, and

2. $V$ is the forgetful functor on operators $\Omega^{\prime}-\Omega^{\prime \prime}$ and identities $E^{\prime}-E^{\prime \prime}$ and $U$ is a functor commuting with the underlying set functors on $\mathscr{A}$ and $\mathscr{B}$. Note that $U$ is not necessarily a functor forgetting part of $\Omega$ and $E$.

We next describe a functor $C_{V}: \mathscr{B} \rightarrow$ Grph associated to each pair consisting of a standard diagram $V U$ of algebras and an adjunction $\left\langle L, V U, \phi^{\prime}\right\rangle: \mathscr{D} \rightarrow \mathscr{A}$, where Grph is the category of directed graphs in the sense of [4]. Given $G \in|\mathscr{B}|$ the objects of the graph $C_{V}(G)$ are the elements of the underlying set $|L V G|$ of $L V G$ and its arrows are described recursively by:

1. $\omega_{U L V G}\left(\left|\eta_{V G}^{\prime}\right| x_{1}, \cdots,\left|\eta_{V G}^{\prime}\right| x_{n}\right) \rightarrow\left|\eta_{V G}^{\prime}\right| \omega_{G}\left(x_{1}, \cdots, x_{n}\right)$ is an arrow if $\omega$ is in the set $\Omega^{\prime}-\Omega^{\prime \prime}$ of operators forgotten by $V$ and $\left|\eta_{V G}^{\prime}\right|:|G| \rightarrow$ $|L V G|$ is the set map underlying the unit $\eta_{V G}^{\prime}: V G \rightarrow V U L V G$ of the adjunction $\left\langle L, V U, \phi^{\prime}\right\rangle$ and $\left(x_{1}, \cdots, x_{n}\right)$ is an $n$-tuple of elements of $|G|$ for which $\omega_{G}\left(x_{1}, \cdots, x_{n}\right)$ is defined.

AMS (MOS) subject classifications (1970). Primary 18B15, 18C10, 08A25; Secondary 17B35, 20E30. 
2. If $d \rightarrow e$ is an arrow of $C_{V}(G)$, then so is $\rho_{L V G}\left(d_{1}, \cdots, d, \cdots, d_{q}\right) \rightarrow$ $\rho_{L V G}\left(d_{1}, \cdots, e, \cdots, d_{q}\right)$ for $\rho$ an operator of arity $q$ in $\Omega$ and $d_{1}, \cdots, d_{2-1}, d_{i+1}, \cdots, d_{q}$ arbitrary elements of $|L V G|$.

If $\beta: G \rightarrow G^{\prime}$ is in $\mathscr{B}$, then $C_{V}(\beta): C_{V}(G) \rightarrow C_{V}\left(G^{\prime}\right)$ is the graph morphism which is the function $|L V \beta|:|L V G| \rightarrow\left|L V G^{\prime}\right|$ on objects and is defined recursively on arrows in the obvious way. The purpose of condition 1 is to make $\eta^{\prime}$ into a morphism for each operator of $\Omega^{\prime}$. The graph is designed to impose an equivalence relation on $|L V G|$, compatible with this requirement on $\eta^{\prime}$, so that the resulting equivalence classes inherit an $\langle\Omega, E\rangle$ algebra structure from $L V G$.

The component class $[X]$ of an object $X$ of a category $\mathscr{C}$ (or a graph $\mathscr{C}$ ) is the class of all objects $Y$ which can be connected to $X$ by a finite sequence of morphisms (e.g. $X \rightarrow X_{1} \leftarrow X_{2} \rightarrow Y$ ). We let Comp $\mathscr{C}$ denote the ccllection of component classes.

In the following theorem note that if $V U$ is a standard diagram with $\mathscr{D}$ the category of sets, then an adjunction $\left\langle L, V U, \phi^{\prime}\right\rangle:$ Sets $\rightarrow \mathscr{A}$ can be described easily by letting $L X$ be the free $\langle\Omega, E\rangle$ algebra on the set $X$.

THEOREM 1. Let $V U: \mathscr{A} \rightarrow \mathscr{B} \rightarrow \mathscr{D}$ be a standard diagram of algebras with given adjunction $\left\langle L, V U, \phi^{\prime}\right\rangle: \mathscr{D} \rightarrow \mathscr{A}$. Then there is an adjunction $\langle F, U, \phi\rangle: \mathscr{B} \rightarrow \mathscr{A}$ with the following specific properties:

(a) The underlying set of $F G$ is $\operatorname{Comp} C_{V}(G)$.

(b) If $\rho$ is an operator of arity $n$ in $\Omega$, then $\rho_{F G}$ is defined by $\rho_{F G}\left(\left[c_{1}\right], \cdots,\left[c_{n}\right]\right)=\left[\rho_{L V G}\left(c_{1}, \cdots, c_{n}\right)\right]$ where $c_{1}, \cdots, c_{n}$ are members of the set $|L V G|$ of objects of the graph $C_{V}(G)$.

(c) The unit morphism $\eta_{G}: G \rightarrow U F G$ of $\langle F, U, \phi\rangle$ has an underlying set map which is the composition [ ] $\left|\eta_{V G}^{\prime}\right|$, where $\left|\eta_{V G}^{\prime}\right|:|G| \rightarrow|L V G|=$ $\mathrm{Ob} C_{V}(G)$ is the set map underlying the unit $\eta_{V G}^{\prime}: V G \rightarrow V U L V G$ of the adjunction $\left\langle L, V U, \phi^{\prime}\right\rangle$ and $\left[\mathrm{]}: \mathrm{Ob} C_{V}(G) \rightarrow \operatorname{Comp} C_{V}(G)\right.$ is the component function.

Suppose that the hypotheses of Theorem 1 hold. Let $S_{V}$ be a subgraph of $C_{V}(G)$ having the same objects $|L V G|$ and the same components as $C_{V}(G)$. Then Theorem 1 clearly remains valid upon substitution of $S_{V}$ for $C_{V}(G)$ throughout. This allows us to "picture" the adjoint using a possibly smaller set of arrows than those present in $C_{V}(G)$. Accordingly we define a $V$ picture of the adjoint $F$ to $U$ at $G \in|\mathscr{B}|$ to be any quotient category $\mathscr{C}$ (in the sense of Mac Lane [4]) of the free category generated by such a subgraph $S_{V}$ of $C_{V}(G)$. Theorem 1 is then valid upon substitution of the underlying graph of a $V$ picture $\mathscr{C}$ for $C_{V}(G)$ throughout. Two distinct examples of such $V$ pictures are given in $\S 3$.

Let $\mathscr{G}$ be a small subcategory of a category $\mathscr{C}$ and let $\mathscr{P}(\mathscr{G})$ be the power category of $\mathscr{G}$. The objects of $\mathscr{P}(\mathscr{G})$ are the subclasses of objects 
of $\mathscr{G}$ and the morphisms are inclusions. The reduction functor $\mathscr{R}_{\mathscr{G}}: \mathscr{C}^{\mathrm{op}} \rightarrow$ $\mathscr{P}(\mathscr{G})$ is defined by

$$
\mathscr{R}_{\mathscr{G}} X=\{A \mid A \text { is an object of } \mathscr{G} \text { and } X \rightarrow A \text { exists in } \mathscr{C}\}
$$

with the obvious definition on morphisms. The subcategory $\mathscr{G}$ is reduced in $\mathscr{C}$ if $\mathscr{R}_{\mathscr{G}} A=\{A\}$ for each $A$ in the class $|\mathscr{G}|$ of $\mathscr{G}$ objects. An object $X$ of $\mathscr{C}$ is $\mathscr{G}$ reducible if $\mathscr{R}_{\mathscr{G}} X$ is nonempty.

THEOREM 2. Let $V U: \mathscr{A} \rightarrow \mathscr{B} \rightarrow \mathscr{D}$ be a standard diagram of algebras with given adjunction $\left\langle L, V U, \phi^{\prime}\right\rangle: \mathscr{D} \rightarrow \mathscr{A}$ and suppose that $\langle F, U, \phi\rangle: \mathscr{B} \rightarrow$ $\mathscr{A}$ is the adjunction described in Theorem 1 . Furthermore let $\mathscr{C}$ be any $V$ picture of the adjoint $F$ to $U$ at $G \in|\mathscr{B}|$. Then the unit morphism $\eta_{G}: G \rightarrow$ $U F G$ of $\langle F, U, \phi\rangle$ is monic if and only if the following hold:

(a) The discrete subcategory $\mathscr{G}=\eta_{V G}^{\prime}(|G|)$ is reduced in $\mathscr{C}$ for $\eta_{V G}^{\prime}$ the unit of $\left\langle L, U V, \phi^{\prime}\right\rangle$.

(b) If $[A]=[B]$ in Comp $\mathscr{C}$ with $A, B \in|\mathscr{G}|$, then $\mathscr{R}_{\mathscr{G}} A=\mathscr{R}_{\mathscr{G}} B$.

(c) The unit morphism $\eta_{V G}^{\prime}$ of $\left\langle L, V U, \phi^{\prime}\right\rangle$ is monic.

2. Sufficient conditions. Let $\mathscr{N}$ be the preorder of nonnegative integers with $n \rightarrow m$ iff $n \geqq m$. A rank functor for a category $\mathscr{C}$ is a functor $R: \mathscr{C} \rightarrow \mathscr{N}$ with $R \alpha \neq 1$ whenever $\alpha \neq 1$. Given an object $X$ of $\mathscr{C}$ let $(X / \mathscr{C})_{\mathscr{P}}$ be the category whose objects are the nonidentity $\mathscr{C}$ morphisms of domain $X$ and whose morphisms $\gamma: \alpha \rightarrow \beta$ are $\mathscr{C}$ morphisms for which $\gamma \alpha=\beta$.

THEOREM 3. Let $\mathscr{C}$ be a category with rank functor given and suppose that $\mathscr{G}$ is a subcategory reduced in $\mathscr{C}$. Then the following are equivalent:

(a) The categories $(X / \mathscr{C})_{\mathscr{P}}$ are connected for each $X \in|\mathscr{C}|$ which is $\mathscr{G}$ reducible.

(b) If $[X]=[Y]$ in Comp $\mathscr{C}$, then $\mathscr{R}_{\mathscr{G}} X=\mathscr{R}_{\mathscr{G}} Y$. Furthermore $\mathscr{C}(X, A)$ has at most one element for each pair $(X, A)$ with $X \in|\mathscr{C}|$ and $A \in|\mathscr{G}|$.

THEOREM 4. Let $V U: \mathscr{A} \rightarrow \mathscr{B} \rightarrow \mathscr{D}$ be a standard diagram of algebras given together with adjunctions $\left\langle L, V U, \phi^{\prime}\right\rangle: \mathscr{D} \rightarrow \mathscr{A}$ and $\langle F, U, \phi\rangle: \mathscr{B} \rightarrow \mathscr{A}$ described as in Theorem 1. Then the unit morphism $\eta_{G}: G \rightarrow U F G$ of $\langle F, U, \phi\rangle$ is monic provided there exists a $V$ picture $\mathscr{C}$ of the adjoint $F$ to $U$ at $G$ for which the following conditions hold:

(a) $\mathscr{C}$ has a rank functor.

(b) The discrete subcategory $\mathscr{G}=\eta_{V G}^{\prime}(|G|)$ is reduced in $\mathscr{C}$ for $\eta_{V G}^{\prime}$ the unit of $\left\langle L, V U, \phi^{\prime}\right\rangle$.

(c) The categories $(X / \mathscr{C})_{\mathscr{P}}$ are connected for each $X \in|\mathscr{C}|$ which is $\mathscr{G}$ reducible.

(d) The unit morphism $\eta_{V G}^{\prime}$ of $\left\langle L, V U, \phi^{\prime}\right\rangle$ is monic. 
3. Applications. A theorem of Schreier states that if

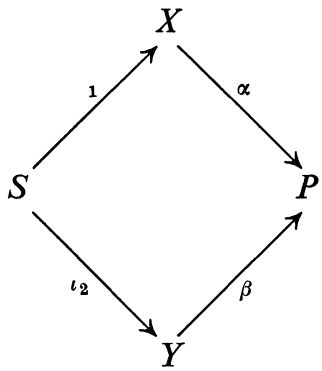

is a pushout diagram in the category of groups with $\iota_{1}$ and $\iota_{2}$ inclusion mappings, then $\alpha$ and $\beta$ are monomorphisms. This may be proved using Theorem 4 as follows. Let $G$ be the disjoint union of the sets underlying $X$ and $Y$ with common subset $S$ identified and $a \cdot b$ defined in $G$ by its $X$ or $Y$ value if both $a, b \in X$ or $a, b \in Y$, otherwise $a \cdot b$ is undefined. Then $G$ is an object of the category $\mathscr{B}$ of sets with a partially defined binary operation. Clearly $\delta \iota_{1}: S \rightarrow X \rightarrow G$ and $\gamma \iota_{2}: S \rightarrow Y \rightarrow G$ are the sides of a pushout diagram in $\mathscr{B}$ for $\delta$ and $\gamma$ the inclusions, and it is sufficient to show $G$ is embeddable in a group. Let $V U: \mathscr{A} \rightarrow \mathscr{B} \rightarrow$ Sets be the standard diagram with $\mathscr{A}$ the category of semigroups and $U$ forgetful. Theorems 1,2 , and 4 remain valid when $\mathscr{B}$ and $\mathscr{D}$ (but not $\mathscr{A}$ ) are categories of partially defined algebras. The graph $C_{V}(G)$ has elements of the free semigroup on $|G|$ as objects and arrows of the form $\left(a_{1}, \cdots, a_{n}\right) \rightarrow\left(a_{1}, \cdots, a_{i}\right.$. $\left.a_{i+1}, \cdots, a_{n}\right)$ for $a_{i} \cdot a_{i+1}$ defined in $G$ and $n>1$. We choose as $V$ picture the preorder $\mathscr{C}$ which is a quotient of the free category on $C_{V}(G)$. The only nontrivial condition of Theorem 4 is the verification that for $X=\left(a_{1}, \cdots\right.$, $\left.a_{n}\right) \mathscr{G}$ reducible, any two $C_{V}(G)$ arrows with domain $X$ considered as objects of $(X / \mathscr{C})_{\mathscr{P}}$ can be connected in $(X / \mathscr{C})_{\mathscr{P}}$. This is true for the particular $G$ described (but not all objects of $\mathscr{B}$ ) and shows $G$ embeddable in a semigroup $F G$, which turns out to be a group from the way $G$ is defined.

The classical Birkhoff Witt theorem states that every Lie algebra $G$ which is free as a $K$ module can be embedded in its universal associative algebra $F G$. This may be proved from Theorem 4 by verifying its conditions for the following $V$ picture $\mathscr{C}$ of the adjoint $F$ to $U$ at $G$, where $V U: \mathscr{A} \rightarrow \mathscr{L} \rightarrow \operatorname{Mod}_{K}$ is the standard diagram with $\mathscr{A}$ and $\mathscr{L}$ the categories of associative and Lie algebras over $K$, respectively, and multiplication $[a, b]=a \cdot b-b \cdot a$ in $U A$ defined from that in $A$. We let $\mathscr{C}$ be the preorder which is a quotient of the free category on the following subgraph $S_{V}$ of $C_{V}(G)$. The objects of $S_{V}$ are the elements of the free $K$ module $L V G$ on all finite strings $x_{i_{1}} \cdots x_{i_{n}}$ of elements from a basis $\left(x_{i}\right)_{i \in I}$ of the free $K$ module $V G$. Given a well ordering of $I$ we let the arrows of $S_{V}$ be those 
of the form

$$
\begin{aligned}
k_{i} x_{i_{1}} \cdots x_{i_{n}}+\alpha \rightarrow & k_{i} x_{i_{1}} \cdots x_{i_{j+1}} x_{i_{j}} \cdots x_{i_{n}} \\
& +k_{i} x_{i_{1}} \cdots\left[x_{i_{j}}, x_{i_{j+1}}\right] \cdots x_{i_{n}}+\alpha
\end{aligned}
$$

for $i_{j+1}<i_{j}, k_{i} \in K$, and $\alpha$ any element of $L V G$ (not involving $x_{i_{1}} \cdots x_{i_{n}}$ ). The crucial step of Theorem 4 is the verification that $(X / \mathscr{C})_{\mathscr{P}}$ is connected for each $\mathscr{G}$ reducible object $X$ of $\mathscr{C}$. This comes down to showing that for $c<b<a$ in $I$ the objects

and

$$
\beta: x_{a} x_{b} x_{c} \rightarrow x_{b} x_{a} x_{c}+\left[x_{a}, x_{b}\right] x_{c}
$$

$$
\gamma: x_{a} x_{b} x_{c} \rightarrow x_{a} x_{c} x_{b}+x_{a}\left[x_{b}, x_{c}\right]
$$

can be connected in $\left(\left(x_{a} x_{b} x_{c}\right) / \mathscr{C}\right)_{\mathscr{p} \text {. }}$. This is done by further reduction of the ranges of $\beta$ and $\gamma$ and use of Jacobi identity and the identity $[x, y]=-[y, x]$. Details will appear elsewhere.

\section{REFERENCES}

1. R. Baer, Free sums of groups and their generalizations. An analysis of the associative law, Amer. J. Math. 71 (1949), 706-742. MR 11, 78.

2. G. Bergman, The diamond lemma for ring theory (preprint).

3. G. Birkhoff, Representability of Lie algebras and Lie groups by matrices, Ann. of Math. (2) 38 (1937), 526-532.

4. S. Mac Lane, Categories for the working mathematician, Springer-Verlag, New York and Berlin, 1971.

5. H. Neumann, Generalized free products with amalgamated subgroups, Amer. J. Math. 70 (1948), 590-625. MR 10, 233.

6. M. H. A. Newman, On theories with a combinatorial definition of "equivalence", Ann. of Math. (2) 43 (1942), 223-243. MR 4, 126.

7. J. Serre, Lie algebras and Lie groups, Benjamin, New York, 1965. MR 35 \#6721.

Department of Mathematics, University of British Columbia, Vancouver 8, CANADA 